\title{
25 Research Soure \\ Identification of Type 2-pediatric Asthma Based on Single-cell Transcriptomic Analysis
}

\section{Bing Dai}

Shengjing Hospital of China Medical University

\section{Feifei Sun}

Shengjing Hospital of China Medical University

\section{Nan Yang}

Shengjing Hospital of China Medical University

Xuxu Cai

Shengjing Hospital of China Medical University

\section{Chunlu Li}

Shengjing Hospital of China Medical University

Yunxiao Shang ( $D$ sxy15088706914@163.com )

Shengjing Hospital of China Medical University https://orcid.org/0000-0002-8885-4937

\section{Research Article}

Keywords: Type 2-pediatric asthma, single-cell sequence, transcriptomic analysis, immune cells, consensus clustering

Posted Date: June 29th, 2021

DOl: https://doi.org/10.21203/rs.3.rs-637521/v1

License: (c) (i) This work is licensed under a Creative Commons Attribution 4.0 International License.

Read Full License 


\section{Abstract}

Type 2-pediatric asthma characterized by T2 cytokine-driven airway inflammation is the most common type of asthma. Recently, T2 cytokine inhibitors have reduced the exacerbation rates of asthma, but their ability to improve lung function is limited. Screening novel therapeutic strategies for Type 2-pediatric asthma patients is imperative. We obtained single-cell RNA sequencing (scRNA-seq) describing the chronic stimulation GSE145013 dataset with IL-13. Consensus clustering was performed to classify pediatric asthmatic patients from validation datasets GSE65204 and GSE40888, based on the cell marker genes. We found three cellular subtypes including ciliated cells, secretory cell 1 , and secretory cell 2 . The expression of CCL26, PRB1, and SLC9B2 was higher in secretory cell 1, while SCGB3A1 and BPIFA1 were higher in secretory cell 2 . Consensus clustering based on the five cell marker genes produced two patient subtypes (C1 and C2). The expression of SCGB3A1 and BPIFA1 was higher in C2 subtypes, while CCL26, $P R B 1$, and $S L C 9 B 2$ was higher in $\mathrm{C} 1$ subtypes. Patients in $\mathrm{C} 2$ subtypes may more secretory cell 2, while the patients in $\mathrm{C} 1$ may have higher secretory cell 1 in the infiltrate. More Type $2 \mathrm{~T}$ helper cells were in the infiltrate in the $\mathrm{C} 2$ subtype, while type $1 \mathrm{~T}$ helper cells were higher in the $\mathrm{C} 1$ subtype. T2 cytokines (IL-13, IL-33, IL-3, IL-4, and TSLP) were expressed more in the C2 subtype, corresponding to Type 2-pediatric asthma. This study identified five cell marker genes to screen Type 2-pediatric asthma that could potentially be therapeutic targets for Type 2-pediatric asthma.

\section{Introduction}

Bronchial asthma is a common respiratory disease in children, mainly related to environmental and genetic factors. It is more common in spring and autumn owing to increased airway reactivity, resulting in airway constriction [1]. Type 2-pediatric asthma as the most common type of asthma, is characterized by infiltration of inflammatory cells in the lung tissue, including eosinophils, mast cells, lymphocytes, accompanied by excessive differentiation of Type $2 \mathrm{~T}$ helper cells. The infiltrating Type $2 \mathrm{~T}$ helper cells secrete cytokines (IL-4, IL-5, and IL-13), which initiate and exacerbate allergic inflammation [2]. Recently, T2 cytokine inhibitors have been shown to reduce the exacerbation rates of Type 2-pediatric asthma, but their ability to improve lung function is limited. Therefore, further study on the mechanism of Type 2pediatric asthma at the molecular level and the search for biomarkers related to Type 2-pediatric asthma will be beneficial to the development of new targeted drugs for Type 2-pediatric asthma.

Single-cell RNA sequencing (scRNA-seq) is a new technology for the amplification and sequencing of the whole transcriptome at the single-cell level and can obtain the mRNA expression information of each cell and determine different cell subsets [3]. ScRNA-seq has been used in the study of asthma, and some progress has been made. Tibbitt et al. [4] revealed a novel gene expression signature of airway epithelium Th2 cells in asthma patients, which provides a basis for the study of the mechanism of Th2 cells involved in asthma. Jiang et al. [5] screened specific genes to characterize and quantify mast cells in asthma using scRNA-sEq. Ailu Chen et al.[6] identified six main cell subsets in severe asthma (SA), containing $\mathrm{CD} 4^{+} \mathrm{T}$ cells, natural killer (NK) cells, $\mathrm{CD} 8^{+} \mathrm{T}$ cells, dendritic cells (DCs), B cells, and monocytes by scRNA-sEq. Among them, $\mathrm{CD} 4^{+} \mathrm{T}$ cells were the dominant cell subtype in SA. In addition, 
they also found that SA patients have a strong response to the stimulation of polyinosine-polycytidylic acid (poly I:C). Therefore, sRNA-seq data can help us to dissect the cells and genes that play a key role in the development of asthma.

In our study, to reveal the mechanism and find new therapeutic strategies for Type 2-pediatric asthma, we obtained the scRNA-seq data describing chronic stimulation with IL-13 from the Gene Expression Omnibus (GEO) database. Seurat software was used for cell cluster analysis and produced three cellular subtypes, including ciliated cells, secretory cell 1 , and secretory cell 2 . The expression of cell marker genes CCL26, PRB1, SLC9B2, SCGB3A1, and BPIFA1 were reversed in secretory cell 1 and secretory cell 2. Consensus clustering was performed based on the five cell marker genes in the validation dataset and produced two patient subtypes ( $\mathrm{C} 1$ and $\mathrm{C} 2$ ). Type $2 \mathrm{~T}$ helper cells were higher in the infiltrate in the $\mathrm{C} 2$ subtype, while type $1 \mathrm{~T}$ helper cells were higher in the infiltrate in the C1 subtype. T2 cytokines (IL-13, IL33 , IL-3, IL-4, and TSLP) were expressed more in the C2 subtype. We hypothesized that the patients in the C2 subtype corresponded to type 2-pediatric asthma.

\section{Materials And Methods}

\subsection{Data acquisition}

We downloaded the pediatric asthma scRNA-seq describing chronic stimulation with IL-13 in the GSE145013 dataset from the GEO database (https://www.ncbi.nlm.nih.gov/geo/query/acc.cgi? acc=GSE145013) [7]. Single cell sequencing analysis was performed on epithelial cells from tracheal donors after chronic ( 11 days) IL-13 stimulation. A total of 802 single cell data were obtained. In addition, we obtained the transcriptome expression profiles of GSE65204 [8], and GSE40888 [9] datasets containing 36 and 65 pediatric asthma patients, respectively, from the GEO database.

\subsection{Data processing}

The 'Seurat' package was used to process the scRNA-seq data. The PercentageFeatureset function was used to calculate the percentage of mitochondrial genes. Correlation analysis was used to analyze the relationship between sequencing depth and mitochondrial gene sequences, and/or intracellular total sequences. We then removed the cells with mitochondrial gene content $>5 \%$, sequencing number $<50$, and gene number $<100$. Variance analysis was used to screened the first 1500 genes with highly variable characteristics [10].

\subsection{Dimensionality reduction and cell annotation}

Principal Component Analysis (PCA) was used for dimensionality reduction of the sRNA-seq data. Cluster analysis was performed using the $t$-distributed stochastic neighbor embedding ( $t$-SNE) algorithm based on the principal components (PCs) with $p<0.05$. In addition, $P<0.05$, and $\log _{2}|F C|>0.5$ were used as cut- 
off criteria to screen out marker genes in each cluster group. The top 10\% marker genes in the cluster were listed in the heat map. The "Singler" package was performed for clustering annotation according to the marker genes $[11,12]$. The clusterProfiler package was performed for Gene Ontology (G0) enrichment analysis based on the marker genes of each cluster. Finally, the "Monocle" algorithm was carried out for pseudotime and trajectory analysis [13].

\subsection{Consensus clustering algorithm based on the cell marker genes}

The "ConsensusClusterPlus" package was used to classify pediatric asthmatic patients in the validation datasets (GSE65204 and GSE40888) based on the selected cell marker genes and 50 repetitions with maxK $=9$ were performed to ensure stable subtypes [14].

\subsection{Single-sample gene set enrichment analysis}

The "GSVA" "limma" and "GSEABase" packages were used to calculate the abundance of the 28 Tumor infiltrating immune cells (TIICs) types in pediatric asthmatic patients from the GSE65204 and GSE40888 datasets. We searched for the marker genes of each immune cell type in the transcriptome expression profiles of patients with pediatric asthma and calculated the abundance of the 28 immune cell types in each sample based on the expression levels of the marker genes.

\subsection{Statistical analysis}

The Wilcoxon rank sum test was used to determine whether the difference between two groups was statistically significant. All statistical analyses were performed in R Studio (version 4.0.3) software, and $P$ $<0.05$ was used to indicate statistically significant differences.

\section{Results}

\subsection{Quality control and normalization of scRNA-seq data}

The scRNA-seq data were qualitatively controlled using the 'Seurat' package, and the number of genes in a cell (nFeature_RNA), transcription number (nCont_RNA), and mitochondrial gene ratio (percent.mt) of the cells in the samples were calculated (Figure 1A). After processing, 756 single cells were selected from 802 cells. There was no correlation between nCont_RNA and percent.mt (Figure 1B), while nCont_RNA and $n$ Feature_RNA had a strong positive correlation $(R=0.94$, Figure $1 C)$. A total of 1500 genes with high variation were extracted from 31920 genes (Figure 1D). 


\subsection{Dimensionality reduction and cell annotation}

Dimensionality reduction of the scRNA-seq data using the PCA algorithm indicated that the epithelial cells had clearly separated (Figure 1E). The six PCs with $p<0.05$ were used for further analysis (Figure $1 \mathrm{~F}$ ). The $t-S N E$ algorithm divides the epithelial cells into three clusters (Figure 2B), including ciliated cells, secretory cell 1 , and secretory cell 2 (Figure $2 \mathrm{C}$ ). Differential analysis between the three clusters identified 121 cell marker genes (Supplementary Table 1) and the top 10\% cell marker genes of each cluster were showed by heat map (Figure 2A). The expression of CCL26, PRB1, and SLC9B2 marker genes were higher in secretory cell 1, while SCGB3A1 and BPIFA1 were higher in secretory cell 2 (Figure 3). Pseudotime and trajectory analysis revealed that the ciliated cells could gradually differentiate into secretory cell 1 and secretory cell 2 under the action of IL-3 (Figure 2D and E). Figure 2F indicates that there are three transition cell types before the ciliated cells differentiate into secretory cell 1 and secretory cell 2 . To investigate the functions of the ciliated cells, secretory cell 1 and secretory cell 2, GO enrichment analysis based on the marker genes was performed. The function of secretory cell 1 may be related to glutathione derivative biosynthetic and metabolic process (Figure 4A). Secretory cell 2 may be related to cytokine activity, cation antiporter activity, proton antiporter activity, and chemokine receptor binding (Figure 4B). Ciliated cells may be related to cilium movement and microtubule movement (Figure 4C).

\subsection{Consensus clustering based on the five cell marker genes}

Considering that the expression of cell marker genes CCL26, PRB1, SLC9B2, SCGB3A1, and BPIFA1 were reversed in secretory cell 1 and secretory cell 2 , we performed consensus clustering based on the five cell marker genes to investigate whether the asthma patients could be classified. We firstly downloaded the transcriptome expression profiles of the GSE65204 and GSE40888 datasets from the GEO database and extracted the five cell marker genes. Then, consensus clustering algorithm was performed, and two asthma subtypes (C1 and C2) were obtained (Figure 5A-5C, Figure 6A-6C). The PCA analysis indicated that the asthma patients could be completely separated based on the expression of the five cell marker genes (Figure 5D, Figure 6D). We found that the expression level of SCGB3A1 and BPIFA1 were higher in the $C 2$ subtypes, while $C C L 26, P R B 1$, and $S L C 9 B 2$ were higher in the $C 1$ subtypes (Figure 5E, Figure 6E). We suggest that type $2 \mathrm{~T}$ helper cells were higher in the infiltrate in the $\mathrm{C} 2$ subtype, while type $1 \mathrm{~T}$ helper cells were higher in the infiltrate in the $\mathrm{C} 1$ subtype. It is known that chronic IL-3 stimulation can produce a Type 2-asthma model [7]. We hypothesized that one of the two secretory cells (secretory cell 1 and secretory cell 2) may be associated with Th2 inflammation. To verify our hypothesis, we compared the expression of T2 cytokines and the abundance of immune cell infiltration between the asthma subtypes. We found that the T2 cytokines (IL-13, IL-33, IL-3, IL-4, and TSLP) were highly expressed in the C2 subtype (Figure 5F, Figure 6F). Type $2 \mathrm{~T}$ helper cells were higher in the infiltrate in the $\mathrm{C} 2$ subtype, while type $1 \mathrm{~T}$ helper cells were higher in the infiltrate in the $\mathrm{C} 1$ subtype (Figure 5G, Figure 6G). Thus, we speculate that 
the patients in the $\mathrm{C} 2$ subtype correspond to type 2-pediatric asthma, and secretory cell 2 is associated with Th2 inflammation.

\section{Discussion}

Asthma is a heterogeneous disease, and transcriptome sequencing is often used to study the pathogenesis of asthma and the efficacy of drug intervention in asthma [15-17]. Owing to the heterogeneity of asthma, traditional transcriptome sequencing methods based on blood or tissue samples obtain macroscopic results of the cell population, showing the average expression level of the same gene in all cells, rather than the differences between individual cells of the same gene and its role in the pathogenesis of the disease. Therefore, the pathogenesis of heterogeneous asthma and the therapeutic effect of drugs cannot be completely revealed. ScRNA-seq is a rapidly developing frontier technology in life science in recent years and can reveal the gene expression status of cells at the singlecell level, reflect the heterogeneity between cells, and provide a new research idea and technical means for the study of heterogeneous diseases [18-20]. This study explored the pathogenesis of Type 2-pediatric asthma based on single-cell sequencing data and provides a direction for finding novel targeted therapies.

Asthma affects health of all age groups, and its prevalence is increasing year by year, especially in children. Asthma has different disease processes and can be divided into different asthma phenotypes according to the patient's history, clinical manifestations, pathophysiological characteristics, treatment feedback, and prognostic difference [21, 22]. Lymphocytes play an important role in chronic inflammation in asthma, and the enhanced immune response of type 2 helper T cells (Th2) (type 2 immune response) is considered an important inflammatory phenotype in asthma [23]. At the initial contact with the allergen, epithelial cells detect the allergen, release pro-inflammatory factors, and promote the recruitment and activation of lung dendritic cells, which absorb the allergen and transport the allergen to reflux lymph nodes to induce the activation of allergen-specific Th2 cells. When exposed to the same allergen again, Th2 effector cells are stimulated and activated to secrete type 2 inflammatory factors such as IL-4, IL-5, and IL-13, causing allergic airway inflammation [24]. Currently, T2 cytokine inhibitors reduced the exacerbation rates of asthma, but their ability to improve lung function is limited. Therefore, we need to explore the pathogenesis of Type 2-pediatric asthma at the molecular level to provide a basis for finding new therapeutic targets.

Considering that Type 2-pediatric asthma is an airway obstructive disease driven by IL-13, we obtained an in vitro model of Type 2-pediatric asthma in the GSE145013 dataset, which described the epithelial cells from tracheal donors after chronic (11 days) IL-13 stimulation. Single cell sequencing analysis was performed on the in vitro model and 756 single cells were obtained through quality control and normalization. We then acquired three cell subtypes (ciliated cells, secretory cell 1 , and secretory cell 2 ) and the cell marker genes (CCL26, PRB1, SLC9B2, SCGB3A1, and BPIFA1) were reverse expressed in secretory cell 1 and secretory cell 2 . We suspect that the five cell marker genes may be involved in the pathogenesis of Type 2-pediatric asthma. Consensus clustering was performed based on the five cell 
marker genes in the validation dataset and produced two patient subtypes ( $\mathrm{C} 1$ and $\mathrm{C} 2$ ). Type $2 \mathrm{~T}$ helper cells were higher in the infiltrate in the $\mathrm{C} 2$ subtype, while type $1 \mathrm{~T}$ helper cells were higher in the infiltrate in the $\mathrm{C} 1$ subtype. T2 cytokines (IL-13, IL-33, IL-3, IL-4, and TSLP) were expressed more in the C2 subtype. We speculate that the patients in the $\mathrm{C} 2$ subtype correspond to Type 2-pediatric asthma. According to the above results, we have reason to believe that the five cell marker genes are involved in the pathogenesis of Type 2-pediatric asthma and may be new therapeutic targets for Type 2-pediatric asthma in the future.

\section{Conclusions}

In conclusion, our research found five cell marker genes involved in the pathogenesis of Type 2-pediatric asthma based on scRNA-seq data and transcriptome expression profiles through a series of bioinformatics analysis methods, which provide new direction for precise treatment of Type 2-pediatric asthma. Our study was based on a public database, and further in vitro and in vivo studies are needed to verify the results.

\section{Declarations}

Ethics approval and consent to participate

Not Applicable

Consent for publication

Not Applicable

Availability of data and materials

All data generated or analyzed during this study are included in this published article and its supplementary information files

Competing interests

The authors declare that they have no competing interests

Author Contributions

Bing Dai, Feifei Sun, Xuxu Cai, Chunlu Li, Nan Yang, Yunxiao Shang conceived and designed the study. Bing Dai, Feifei Sun Yunxiao Shang developed the methodology. Bing Dai, Feifei Sun, Xuxu Cai, and Yunxiao Shang analyzed and interpreted the data. Bing Dai, Nan Yang, and Yunxiao Shang wrote, reviewed, and/or revised the manuscript.

Funding 
This work was supported by: Young Scientists Fund of the National Natural Science Foundation of China (No: 81901763), 2018 Liaoning Provincial Natural Science Key Project of China (No: 20180530064), 345 Talent Project from Shengjing Hospital of China Medical University (No: M0282), and 345 Talent Project of Shengjing Hospital of China Medical University (No: M0421), the University Innovation Team of Liaoning Province in 2018.

Acknowledgments

We thank the authors who provided the GEO public datasets.

\section{References}

1. Ramphul M, Lo DKH, Gaillard EA: Precision Medicine for Paediatric Severe Asthma: Current Status and Future Direction. J Asthma Allergy 2021, 14:525-538.

2. Inada T, Kubo K, Shingu K: Promotion of interferon-gamma production by natural killer cells via suppression of murine peritoneal macrophage prostaglandin $\mathrm{E} \mathbb{X}$ production using intravenous anesthetic propofol. Int Immunopharmacol 2010, 10(10):1200-1208.

3. Hwang B, Lee JH, Bang D: Single-cell RNA sequencing technologies and bioinformatics pipelines. Exp Mol Med 2018, 50(8):1-14.

4. Tibbitt CA, Stark JM, Martens L, Ma J, Mold JE, Deswarte K, Oliynyk G, Feng X, Lambrecht BN, De Bleser P et al: Single-Cell RNA Sequencing of the T Helper Cell Response to House Dust Mites Defines a Distinct Gene Expression Signature in Airway Th2 Cells. Immunity 2019, 51(1):169-184.e165.

5. Jiang J, Faiz A, Berg M, Carpaij OA, Vermeulen CJ, Brouwer S, Hesse L, Teichmann SA, Ten Hacken NHT, Timens W et al: Gene signatures from scRNA-seq accurately quantify mast cells in biopsies in asthma. Clin Exp Allergy 2020, 50(12):1428-1431.

6. Chen A, Diaz-Soto MP, Sanmamed MF, Adams T, Schupp JC, Gupta A, Britto C, Sauler M, Yan X, Liu Q et al: Single-cell characterization of a model of poly l:C-stimulated peripheral blood mononuclear cells in severe asthma. Respir Res 2021, 22(1):122.

7. Jackson ND, Everman JL, Chioccioli M, Feriani L, Goldfarbmuren KC, Sajuthi SP, Rios CL, Powell R, Armstrong M, Gomez J et al: Single-Cell and Population Transcriptomics Reveal Pan-epithelial Remodeling in Type 2-High Asthma. Cell Rep 2020, 32(1):107872.

8. Yang IV, Pedersen BS, Liu AH, O'Connor GT, Pillai D, Kattan M, Misiak RT, Gruchalla R, Szefler SJ, Khurana Hershey GK et al: The nasal methylome and childhood atopic asthma. J Allergy Clin Immunol 2017, 139(5):1478-1488.

9. Raedler D, Ballenberger N, Klucker E, Böck A, Otto R, Prazeres da Costa O, Holst O, Illig T, Buch T, von Mutius $\mathrm{E}$ et al: Identification of novel immune phenotypes for allergic and nonallergic childhood 
asthma. J Allergy Clin Immunol 2015, 135(1):81-91.

10. Xiang R, Rong Y, Ge Y, Song W, Ren J, Fu T: Cell differentiation trajectory predicts patient potential immunotherapy response and prognosis in gastric cancer. Aging (Albany NY) 2021, 13(4):59285945.

11. Lall S, Sinha D, Bandyopadhyay S, Sengupta D: Structure-Aware Principal Component Analysis for Single-Cell RNA-seq Data. J Comput Biol 2018.

12. Satija R, Farrell JA, Gennert D, Schier AF, Regev A: Spatial reconstruction of single-cell gene expression data. Nat Biotechnol 2015, 33(5):495-502.

13. Qiu X, Mao Q, Tang Y, Wang L, Chawla R, Pliner HA, Trapnell C: Reversed graph embedding resolves complex single-cell trajectories. Nat Methods 2017, 14(10):979-982.

14. Wilkerson MD, Hayes DN: ConsensusClusterPlus: a class discovery tool with confidence assessments and item tracking. Bioinformatics 2010, 26(12):1572-1573.

15. Bateman ED, Hurd SS, Barnes PJ, Bousquet J, Drazen JM, FitzGerald JM, Gibson P, Ohta K, O'Byrne P, Pedersen SE et al: Global strategy for asthma management and prevention: GINA executive summary. Eur Respir J 2008, 31(1):143-178.

16. Turturice BA, Theorell J, Koenig MD, Tussing-Humphreys L, Gold DR, Litonjua AA, Oken E, RifasShiman SL, Perkins DL, Finn PW: Perinatal granulopoiesis and risk of pediatric asthma. Elife 2021, 10.

17. Dai B, Sun F, Cai X, Li C, Liu H, Shang Y: Significance of RNA N6-Methyladenosine Regulators in the Diagnosis and Subtype Classification of Childhood Asthma Using the Gene Expression Omnibus Database. Frontiers in genetics 2021, 12:634162.

18. Zhao G, Lu H, Liu Y, Zhao Y, Zhu T, Garcia-Barrio MT, Chen YE, Zhang J: Single-Cell

Transcriptomics Reveals Endothelial Plasticity During Diabetic Atherogenesis. Front Cell Dev Bio/2021, 9:689469.

19. Riemondy KA, Venkataraman S, Willard N, Nellan A, Sanford B, Griesinger AM, Amani V, Mitra S, Hankinson TC, Handler MH et al: Neoplastic and immune single cell transcriptomics define subgroupspecific intra-tumoral heterogeneity of childhood medulloblastoma. Neuro Oncol 2021.

20. Obradovic A, Chowdhury N, Haake SM, Ager C, Wang V, Vlahos L, Guo XV, Aggen DH, Rathmell WK, Jonasch E et al: Single-cell protein activity analysis identifies recurrence-associated renal tumor macrophages. Cell 2021, 184(11):2988-3005.e2916.

21. Chupp GL, Kaur R, Mainardi A: New Therapies for Emerging Endotypes of Asthma. Annu Rev Med 2020, 71:289-302. 
22. Fuhlbrigge AL, Castro M: Precision Medicine in Asthma-Using Phenotypes to Understand Endotypes That Lead Us to New Therapeutic Options. J Allergy Clin Immunol Pract 2020, 8(2):496-497.

23. Lloyd CM, Snelgrove RJ: Type 2 immunity: Expanding our view. Sci Immunol 2018, 3(25).

24. Ekstedt S, Stenberg H, Tufvesson E, Diamant Z, Bjermer L, Kumlien Georén S, Cardell LO: The potential role of CD16(high) CD62L(dim) neutrophils in the allergic asthma. Allergy 2019, 74(11):22652268.

\section{Figures}
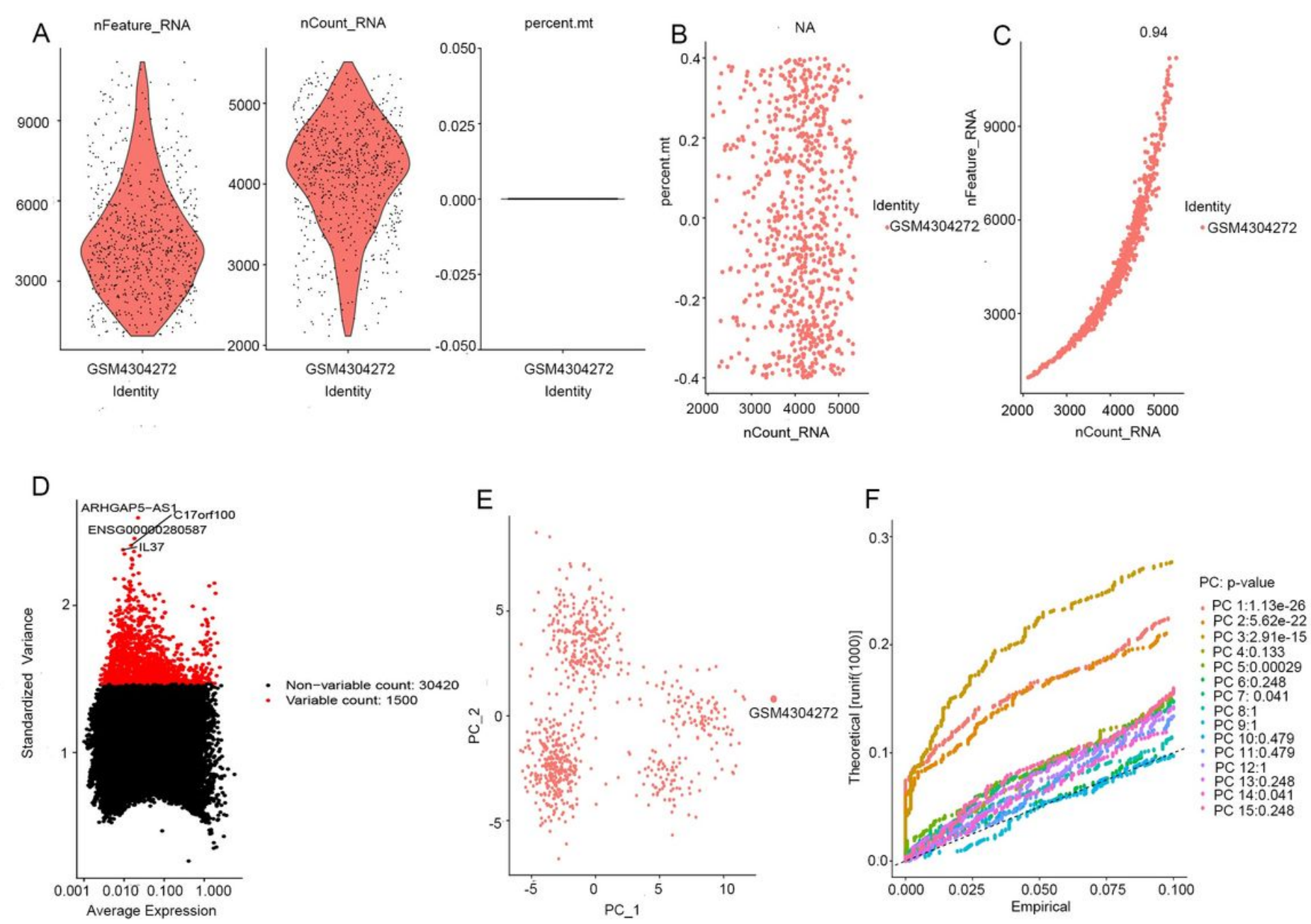

Figure 1

The scRNA-seq data was processed by the 'Seurat' package. (A) Quality control and normalization of scRNA-seq data. (B) Correlation analysis between nCont_RNA and percent.mt. (C) Correlation analysis between nCont_RNA and nFeature_RNA. (D) 1500 genes with high variation from 31920 genes (E) Dimensionality reduction analysis of the scRNA-seq data using PCA algorithm. (F) The principal components (PCs) with $\mathrm{p}<0.05$. 

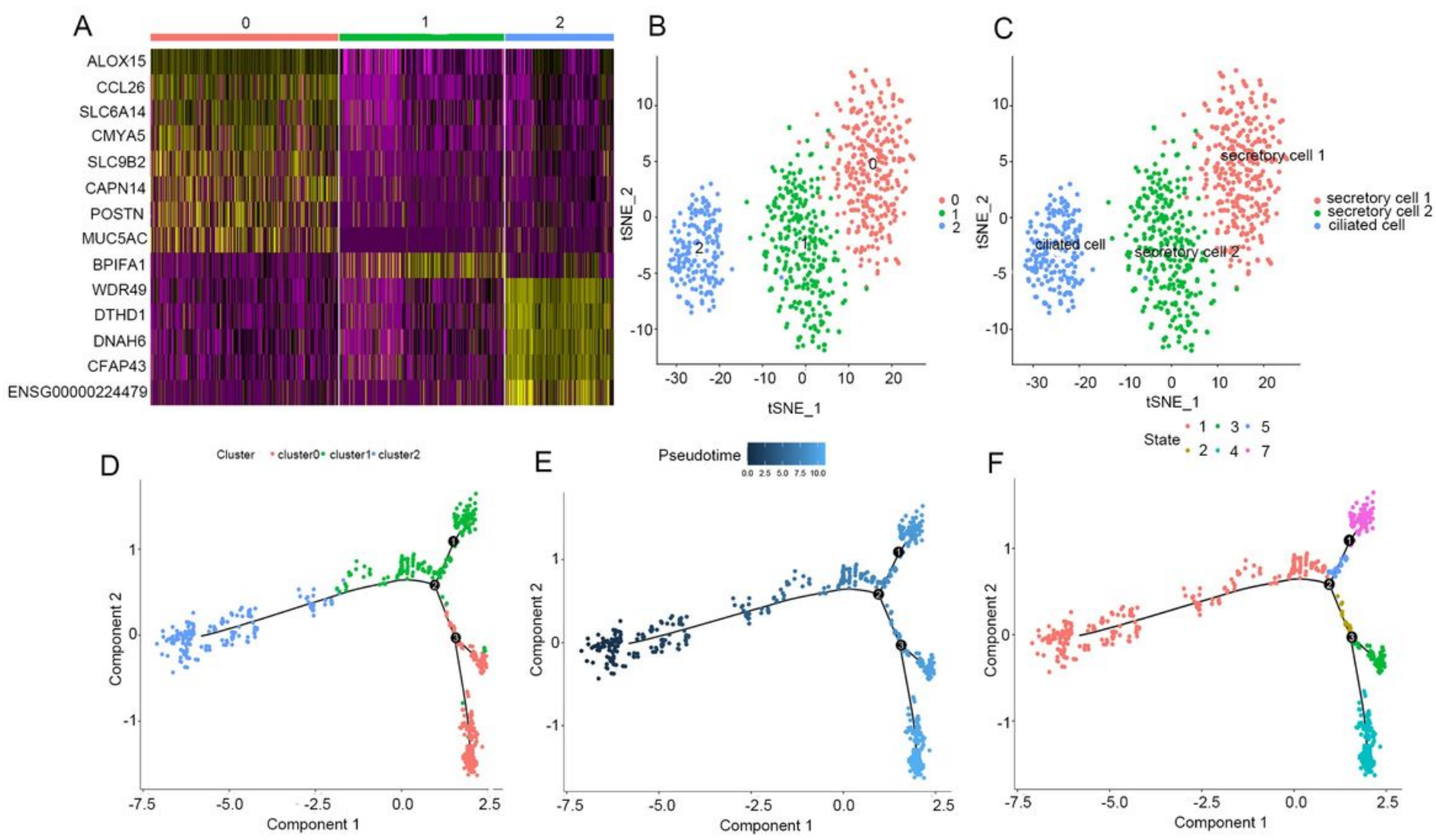

\section{Figure 2}

The scRNA-seq data was analyzed by 'Seurat' package. (A) The top $10 \%$ cell marker genes of each cluster were shown by heat map. (B) The TSNE algorithm divides the epithelial cells into three clusters. (C) Cell annotation of the three cell clusters. (D, E, F) Pseudotime and trajectory analysis. 
CCL26

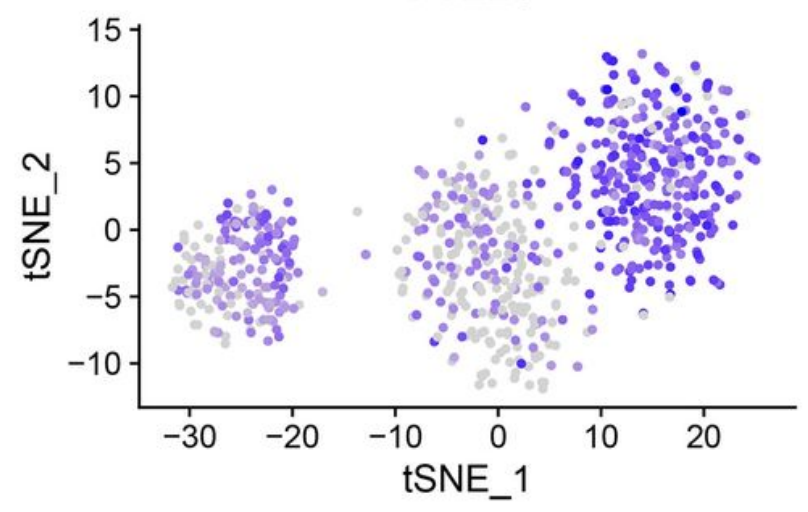

SLC9B2

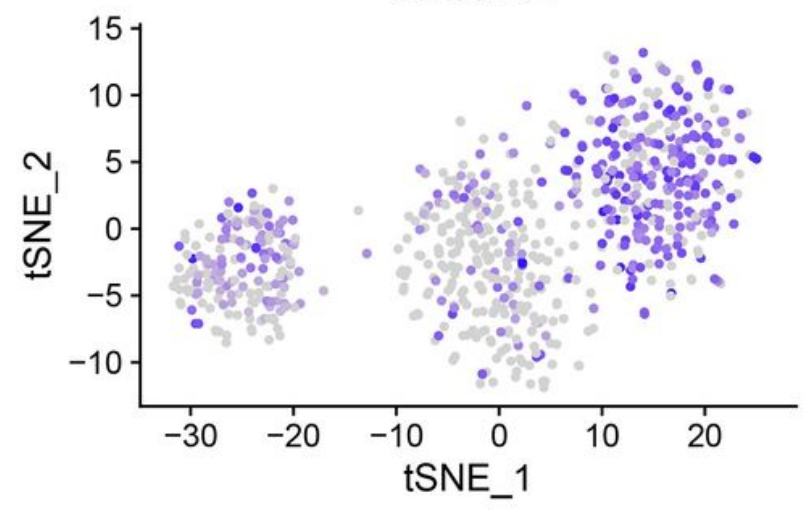

\section{BPIFA1}

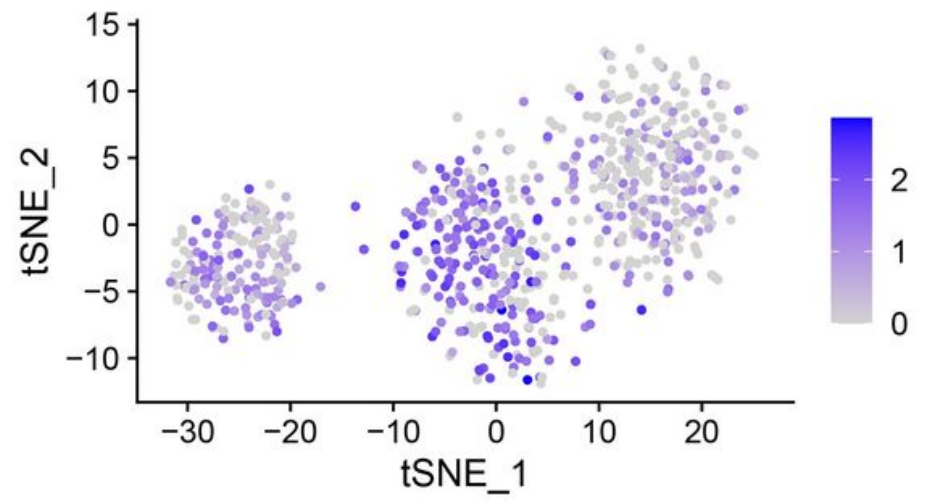

PRB1

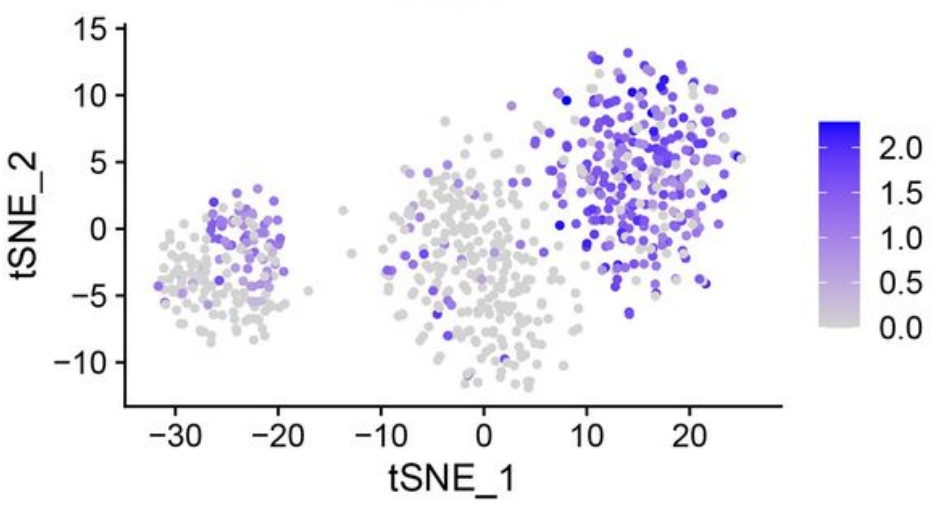

SCGB3A1

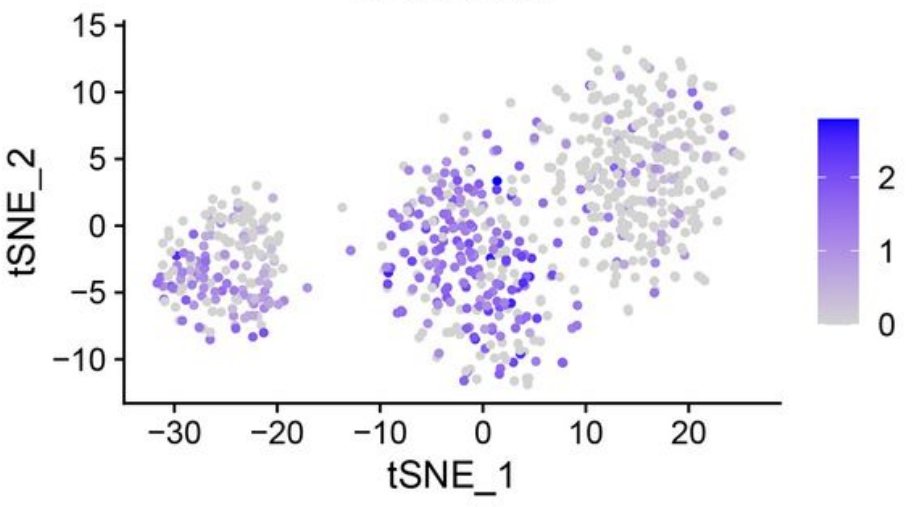

Figure 3

Expression levels of the five cell marker genes (CCL26, PRB1, SLC9B2, SCGB3A1, and BPIFA1) in cell clusters. 


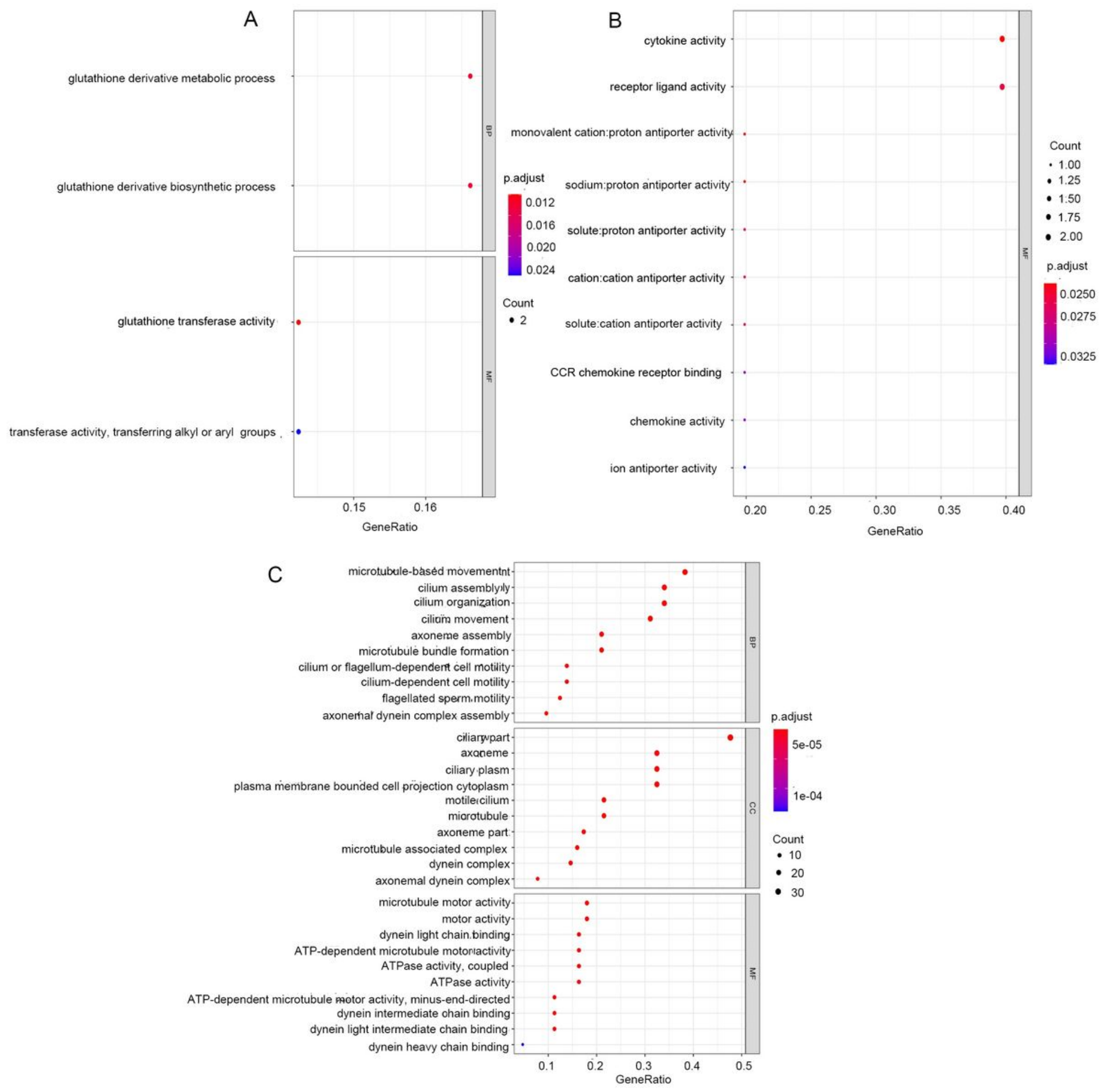

Figure 4

Gene Ontology (GO) enrichment analysis based on the marker genes of each cluster. (A) cluster 0 , (B) cluster 1 , and (C) cluster 2 . 

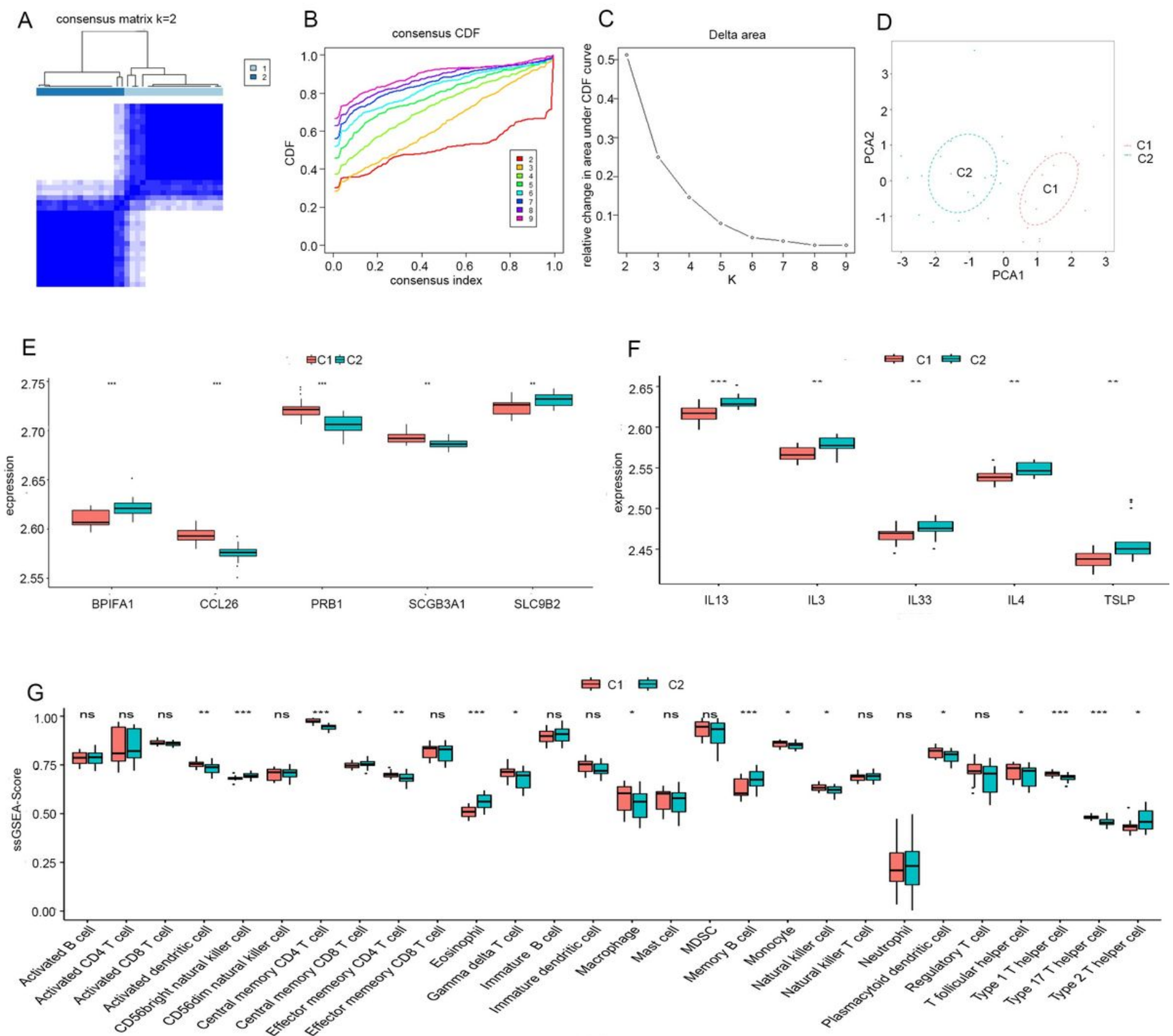

\section{Figure 5}

Consensus clustering algorithm based on the five cell marker genes in the GSE65204 dataset. (A-C) Two patient subtypes were obtained using the consensus clustering algorithm. (D) The PCA analysis indicated that the asthma patients could be completely separated based on the expression of the five cell marker genes. (E) Differential expression of the five cell marker genes in the two patient subtypes. (F) Differential expression of the T2 cytokines in the two patient subtypes. (G) Differential immune cell infiltration in the two patient subtypes. 

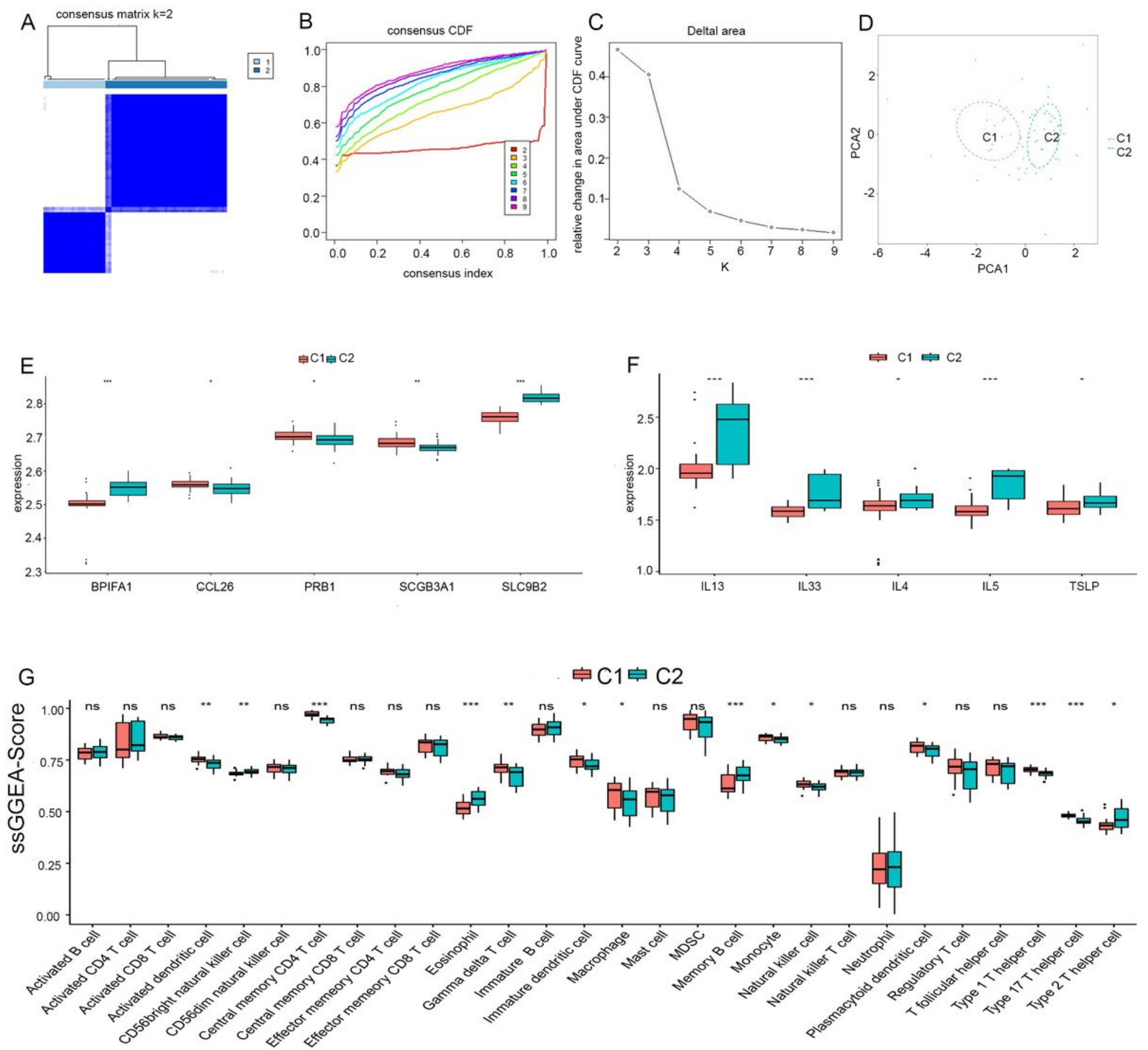

\section{Figure 6}

Consensus clustering algorithm based on the five cell marker genes in the GSE40888 dataset. (A-C) Two patient subtypes were obtained using the consensus clustering algorithm. (D) The PCA analysis indicated that the asthma patients could be completely separated based on the expression of the five cell marker genes. (E) Differential expression levels of the five cell marker genes in the two patient subtypes. (F) Differential expression levels of the T2 cytokines in the two patient subtypes. (G) Differential immune cell infiltration in the two patient subtypes.

\section{Supplementary Files}


This is a list of supplementary files associated with this preprint. Click to download.

- Supplementarytable1.docx 